\title{
Antifungal activity of petroleum ether extracts from Achnatherum inebrians infected with Neotyphodium gansuense
}

\author{
ZHANG XingXu, WU YanPei \& NAN ZhiBiao* \\ State Key Laboratory of Grassland Agro-Ecosystems, College of Pastoral Agriculture Science and Technology, Lanzhou University, \\ Lanzhou 730020, China
}

Received December 9, 2013; accepted April 3, 2014; published online May 19, 2014

Citation: Zhang XX, Wu YP, Nan ZB. Antifungal activity of petroleum ether extracts from Achnatherum inebrians infected with Neotyphodium gansuense. Sci China Life Sci, 2014, 57: 1234-1235, doi: 10.1007/s11427-014-4660-z

Dear Editor,

Achnatherum inebrians (Hance) Keng (drunken horse grass, $\mathrm{DHG})$ is a toxic perennial bunchgrass, which is so-named because it is associated with the narcosis of livestock which graze on native grasslands in the northwest of China [1]. DHG is distributed mainly throughout the harsh conditions of alpine or sub-alpine grasslands, and this species is usually infected by the fungal endophyte Neotyphodium gansuense [2], which apparently enhances its resistance to the abiotic and biotic stress [3].

Endophyte-infected $(\mathrm{E}+)$ or endophyte-free (E-) DHG coarse powders were extracted three times (each for $4 \mathrm{~d}$ ) with $95 \%$ petroleum ether at room temperature. All of the extracts were collected and dried over anhydrous sodium sulphate $\left(\mathrm{Na}_{2} \mathrm{SO}_{4}\right)$, filtered, then rotary evaporated, leaving a faint yellow essential oil which was stored at $4^{\circ} \mathrm{C}$ in sealed, dark-glass vials until required. Drops of these extracts were placed in the center of Petri dishes $(9 \mathrm{~cm}$ diameter) to measure their inhibitory activity on mycelial growth to 17 pathogenic fungi. All dishes were sealed with laboratory film (Parafilm ${ }^{\mathrm{TM}}$, USA). Colony diameters of the pathogens were measured after one week. Each treatment was repeated five times. Inhibition rates were calculated by the formula: $R=[(D c-D p) / D c] \times 100 \%$, where $R$ is inhibition rate, $D c$ is diameter of the control and $D p$ is diameter of the pathogen grown on the PDA with volatiles. Alternaria al-

*Corresponding author (email: zhibiao@1zu.edu.cn) ternata and Bipolaris sorokiniana were isolated from Poa pratensis. Curvularia lunata and Fusarium solani were isolated from A. inebrians. Fusarium avenaceum and Trichoderma viride were isolated from Lolium perenne. Cultures of Colletotrichum gloeosporioides, Fusarium oxysporum (Schl. $f$. sp. niveum), Fusarium oxysporum (f. sp. cubense), Colletotrichum lagenarium, Penicillium italicum and Penicillium digitatum were kindly provided by Professor Gao Kun, College of Chemistry and Chemical Engineering, State Key Laboratory of Applied Organic Chemistry, Lanzhou University. Rhizoctonia solani, Sclerotinia sclerotiorum, Fusarium oxysporum, Alternaria solani, Helminthosporium tritici-vulgaris were gifts from Dr. Zhang ZhenFen, Institute of Microbiology, College of Pastoral Agricultural Science and Technology, Gansu Agricultural University.

Cluster analysis demonstrated that all varieties could be divided into two groups according to their response to the petroleum ether extract of E+ DHG. Specimens were sorted into the "promotion" group, which was further divided into the "strongly promoted" sub-group (P. italicum, B. sorokiniana, $H$. tritici-vulgaris) and the "moderately promoted" sub-group ( $F$. solani and T. viride). The "inhibition group" included twelve varieties, of which $C$. gloeosporioides, $R$. solani, A. solani, C. lunata, F. avenaceum and A. alternata were "most inhibited", while $F$. oxysporum, $P$. digitatum, $S$. sclerotiorum, F. oxysporum Schl. $f$. sp. Niveum, C. lagenarium and F. oxysporum f. sp. Cubense were only "moderately inhibited" (Figure 1A). 

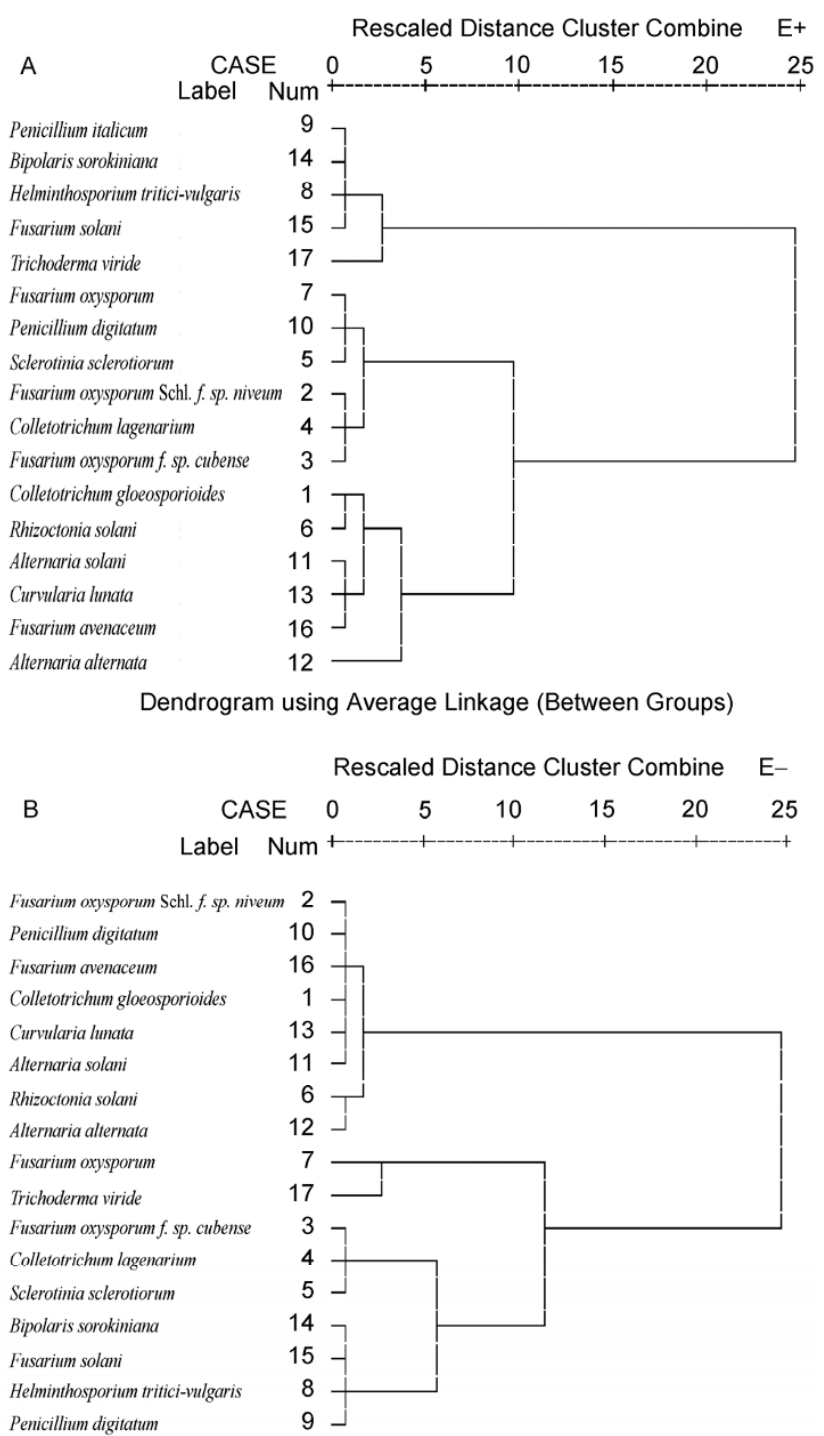

Figure 1 Cluster analysis for inhibition rates of E+ (A) and E- (B) Achnatherum inebrians extracts on the mycelial growth of 17 fungi.

Cluster analysis demonstrated that all varieties could also be divided into two groups according to their response to the petroleum ether extract of E- DHG. Specimens were sorted into the "promotion" group, which was further divided into the "strongly promoted" sub-group (B. sorokiniana, F. solani, $H$. tritici-vulgaris, $P$. Italicum, $F$. oxysporum and $T$. viride) and a "moderately promoted" sub-group ( $F$. oxysporum $f$. sp. cubense, C. lagenarium and S. sclerotiorum). The "inhibition group" included eight varieties, of which $F$. oxysporum Schl. $f$. sp. niveum, $P$. digitatum, $F$. avenaceum, $C$. gloeosporioides, $C$. lagenarium and $A$. solani were "most inhibited", while $R$. solani and A. alternata were only "moderately inhibited" (Figure 1B).
Penicillium italicum, B. sorokiniana, H. tritici-vulgaris, F. solani, T. viride and F. oxysporum were promoted by both the E+ and E- extracts. F. oxysporum Schl. f. sp. Niveum, C. gloeosporioides, R. solani, A. solani, C. lunata, F. avenaceum and A. alternata were inhibited by both the E+ and E- extracts. These extracts differed in their effects on the other fungi. The promotion effect of F. oxysporum was significant $(\mathrm{P}<0.05)$ between the E+ vs. E- extracts, but there was no significant $(\mathrm{P}>0.05)$ difference between the $\mathrm{E}+\mathrm{vs}$. $\mathrm{E}$ - extracts on the other fungi. The inhibition effect of R. solani, F. avenaceum and Alternaria alternata were significant $(\mathrm{P}<0.05)$ between the $\mathrm{E}+\mathrm{vs} . \mathrm{E}-$ extracts, but there was no significant $(\mathrm{P}>0.05)$ difference between the E+ vs. Eextracts for other fungi.

Li et al. [4] reported that the mycelia growth of A. alternata, B. sorokiniana, $C$. lunata and $F$. avenaceum were significantly inhibited by Neotyphodium gansuense. In the present work, the same results were obtained by using crude extract fractions instead of the endophyte directly. Yang et al. [5] reported that the mycelial growth of A. alternata, $B$. sorokiniana, $T$. viride and $F$. avenaceum were obviously inhibited by crude aqueous extracts of E+ vs. E- A. inebrians, and showed that the extract from E+ DHG had the stronger antifungal activity. This evidence implies that $A$. inebrians might eventually be usable as a source of sustainable plant-sourced fungicides, if the relationship between its secondary metabolites, resident endophyte and resistance to pathogens can be clarified by further studies.

We thank Professor Peter Long and Dr. Cory Matthew for polishing the English and giving beneficial discussion of the experiment. This work was supported by the National Basic Research Program of China (2014CB138702) and the Fundamental Research Funds for the Central Universities (Lzujbky-2014-75) and the Program for Changjiang Scholars and Innovative Research Team in University (IRT13019).

1 Li C J, Nan Z B, Gao J H, Tian P. Detection and distribution of Neotyphodium-Achnatherum inebrians association in China. In: Proceedings of 5th International Neotyphodium/Grass Interactions Symposium, Arkansas, USA, 2004. 210

2 Li CJ, Nan ZB, Paul VH, Dapprich P, Liu Y. A new Neotyphodium species symbiotic with drunken horse grass (Achnatherum inebrians) in China. Mycotaxon, 2004, 90: 141-147

3 Zhang XX, Li CJ, Nan ZB, Cory M. Neotyphodium endophyte increases drunken horse grass (Achnatherum inebrians) resistance to herbivores and seed predators. Weed Res, 2012, 52: 70-78

4 Li CJ, Gao JH, Nan ZB. Interactions of Neotyphodium gansuense, Achnatherum inebrians, and plant-pathogenic fungi. Mycol Res, 2007, 111: 1220-1227

5 Yang S, Li CJ, Huang X, Chai Q, Nan ZB. Antifungal activity of acetone extracts of grasses infected with Neotyphodium endophytes (in Chinese). Mycosystema, 2010, 2: 234-240

Open Access This article is distributed under the terms of the Creative Commons Attribution License which permits any use, distribution, and reproduction in any medium, provided the original author(s) and source are credited. 